1 Fundação Oswaldo Cruz (Fiocruz), Escola Nacional de Saúde Pública Sergio

Arouca (Ensp), Programa de Pós-Graduação em Saúde Pública - Rio de Janeiro, Brasil. moraes.veve@gmail.com

2 Fundação Oswaldo Cruz (Fiocruz), Escola Nacional de Saúde Pública Sergio Arouca (Ensp), Programa de Pós-Graduação em Saúde Pública - Rio de Janeiro, Brasil. cristiani@ensp.fiocruz.br

\section{O Programa Bolsa Família e as condicionalidades de saúde: desafios da coordenação intergovernamental e intersetorial}

The Bolsa Família Program and health conditionalities: challenges of intergovernmental and intersectoral coordination

Verena Duarte de Moraes', Cristiani Vieira Machado ${ }^{2}$

RESUMO A existência de condicionalidades de saúde em programas de transferência de renda é controversa e exige coordenação entre áreas sociais. O objetivo do estudo foi discutir as condicionalidades de saúde do Programa Bolsa Família, considerando as diretrizes nacionais, as relações intergovernamentais e intersetoriais. Envolveu revisão bibliográfica, análise documental, análise de dados secundários e realização de 20 entrevistas. Observaram-se fragilidades nas relações entre esferas de governo e setores na gestão do programa. Não houve evidências de que a existência e o acompanhamento das condicionalidades da saúde per se contribuam para o direito à saúde e interrupção do ciclo de pobreza.

PalaVRaS-ChaVe Políticas públicas. Sistema Único de Saúde. Programas governamentais. Pobreza.

ABSTRACT The existence of health conditionalities in income transfer programs is controversial and requires coordination between social areas. The aim of the study was to discuss the health conditionalities of the Bolsa Família Program, considering national guidelines and the intergovernmental and intersectoral relations. It involved literature review, documental analysis, analysis of secondary data and twenty interviews. Frailties have been observed in the relations between levels of government and sectors in the program management. There was no evidence that the existence and monitoring of health conditionalities per se contributes to the right to health and interruption of the cycle of poverty.

KEYWORDS Public policies. Unified Health System. Government programs. Poverty. 


\section{Introdução}

A trajetória histórica da proteção social revela que a expansão da intervenção dos Estados na área social se deu em intensidade e ritmos distintos entre os países desenvolvidos e na região latino-americana (DRAIBE, 1997). Na América Latina (AL), a origem e o desenvolvimento dos sistemas de proteção social apresentam peculiaridades, não se enquadrando nos regimes característicos dos países avançados.

Em geral, as principais características que marcam a origem e a trajetória dos sistemas de proteção social na AL são: desenvolvimento mais tardio em comparação aos países avançados; primeiros programas sociais instituídos nas áreas da previdência e saúde e voltados para categorias profissionais específicas; sistemas erigidos e/ou expandidos sob regimes autoritários e diferenciados entre si, no que concerne à cobertura, variedade, escopo das políticas e resultados sociais (MACHADO, 2012).

A partir dos anos 1980, os processos de globalização, a crise econômica e os programas de ajuste estrutural impulsionados pelas ideias neoliberais provocaram o agravamento da pobreza nessa região (DRAIBE, 2011). Nesse contexto de constrangimentos aos gastos públicos e às políticas sociais abrangentes, os Programas de Transferência de Renda Condicionada (PTRC) surgem na década de 1990 em vários países da AL como uma nova estratégia de política social no combate à pobreza (SIMÕES, 2012).

No Brasil, no contexto de democratização dos anos 1980, a Constituição de 1988 expandiu direitos e inseriu a assistência social no marco abrangente da seguridade, orientada por uma concepção universalista, na contramão das tendências de reforma na AL. Entre as mudanças, destaca-se a instituição de mínimos sociais, como o Benefício de Prestação Continuada (BPC) e a previsão da expansão dos serviços de assistência social. $\mathrm{Na}$ década de 1990, marcada pela liberalização econômica, a implementação das diretrizes constitucionais sofreria diversos percalçOS (MONNERAT, 2009).

Ao final dos anos 1990, os PTRC ganham destaque, no País, na esfera federal, quando são lançados os programas Bolsa Escola, Bolsa Alimentação e Auxílio Gás. Em junho de 2003, com a mudança de governo, foi lançado o Programa Cartão Alimentação. No ano seguinte, ocorreu a unificação desses quatro programas, originando o Programa Bolsa Família (PBF) (BRASIL, 2010).

O PBF possui três pilares centrais: a transferência de renda, as condicionalidades e os programas complementares. A transferência direta de renda tem como objetivo possibilitar alívio imediato da pobreza. As condicionalidades têm como propósito reforçar os direitos à saúde e à educação e, dessa forma, auxiliariam a quebrar o ciclo intergeracional da pobreza. Já as ações e programas complementares visam criar oportunidades para que as famílias superem a situação de vulnerabilidade, proporcionando a melhoria na condição de vida (BRASIL, 2010).

As condicionalidades do programa estão voltadas para a área de educação, saúde e assistência social. Na saúde, as ações compreendem ações básicas, como imunização, pré-natal, acompanhamento do crescimento e desenvolvimento infantil, que já deveriam ser garantidas no âmbito do Sistema Único de Saúde (SUS) (BRASIL, 2010).

O programa brasileiro suscitou, desde sua formulação, posicionamentos contrários e favoráveis no que concerne a sua relevância social e à existência das condicionalidades. Alguns estudiosos questionam que a exigência das condicionalidades às famílias pobres pode-se configurar como uma violação de direitos (LAVINAS, 2013). Já outro grupo de estudiosos relaciona a existência das condicionalidades às questões morais e de juízo de valor, ou seja, ao argumento que ninguém poderia receber um benefício monetário do governo sem dar nada em troca (MEDEIROS; BRITTO; SOARES, 2007).

Somado a esse debate, observa-se a complexidade existente na gestão das 
condicionalidades dado o seu desenho, pois o processo envolve diferentes setores (saúde, educação e assistência), além de incluir diversos atores na sua operacionalização. Nesse sentido, a gestão intersetorial das condicionalidades exige um alto grau de coordenação. Além disso, as relações intergovernamentais constituem desafios no âmbito de um programa de abrangência nacional, com gestão descentralizada, como o PBF. Acrescente-se que governos subnacionais podem ter programas de transferência de renda próprios, bem como propor condicionalidades adicionais para o acesso a essas transferências monetárias.

Em que pese a existência de estudos sobre as condicionalidades da educação, ainda há relativa escassez de pesquisas acerca das condicionalidades de saúde (JANVRY; FINAN; SADOULET, 2007).

Dessa forma, este artigo tem como objetivo discutir as condicionalidades de saúde do PBF, considerando as diretrizes nacionais e as relações intergovernamentais e intersetoriais na gestão das condicionalidades. Para isso, enfocou-se o caso do Rio de Janeiro, em que a relação entre as esferas de governo assume destaque pela existência de um programa municipal - Cartão Família Carioca (CFC) - e um estadual - Renda Melhor (RM) -, além do PBF.

\section{Métodos}

A pesquisa teve caráter predominantemente qualitativo, enfocando a gestão das condicionalidades de saúde do PBF. As dimensões de análise do estudo foram: o desenho nacional das condicionalidades de saúde do PBF; as estratégias de gestão das condicionalidades de saúde adotadas nas esferas nacional, estadual e municipal; a coordenação intergovernamental na gestão das condicionalidades de saúde; a gestão intersetorial das condicionalidades de saúde; e a visão dos dirigentes e profissionais sobre os programas de transferência de renda e as condicionalidades da saúde.
O desenho do estudo previu a análise da gestão das condicionalidades em diferentes escalas (figura 1), dado que a interação entre o desenho nacional da política e sua operacionalização em contextos locais específicos é um aspecto relevante para a compreensão das relações intergovernamentais no PBF. A observação em diferentes escalas também favorece a análise das relações intersetoriais, no caso de políticas sociais de implementação descentralizada.

Para o estudo local, foi escolhido o município do Rio de Janeiro, por apresentar características promissoras para a análise dos desafios de coordenação intersetorial e intergovernamental nas políticas públicas. O município é o segundo mais populoso do País, possui o maior número de beneficiários do PBF no estado do Rio de Janeiro; apresenta uma ampla rede de serviços de saúde e de assistência social, o que é interessante para a análise das relações intersetoriais. Ademais, o município possui um grande número de serviços públicos ligados às diferentes esferas de governo; tem um programa municipal próprio, além de existir um programa estadual com expressão no município, o que o torna um campo rico para a análise das relações intergovernamentais. Ressalte-se que, no momento da pesquisa de campo (realizada em 2014), estado e município eram governados pelo mesmo partido político (Partido do Movimento Democrático Brasileiro PMDB), por sua vez integrantes da coalizão de apoio à Presidência da República (sob governo do Partido dos Trabalhadores PT). Essa situação de 'alinhamento político' também favoreceu a escolha do caso, pois, a princípio, conflitos político-partidários não estariam prejudicando a coordenação intergovernamental.

No município do Rio de Janeiro, a pesquisa de campo foi realizada na área de planejamento que compreende o maior número de beneficiários do programa, apresentando altos índices de pobreza (Área de Planejamento 3.1 - AP 3.1). 
Figura 1. Sistematização do desenho de estudo

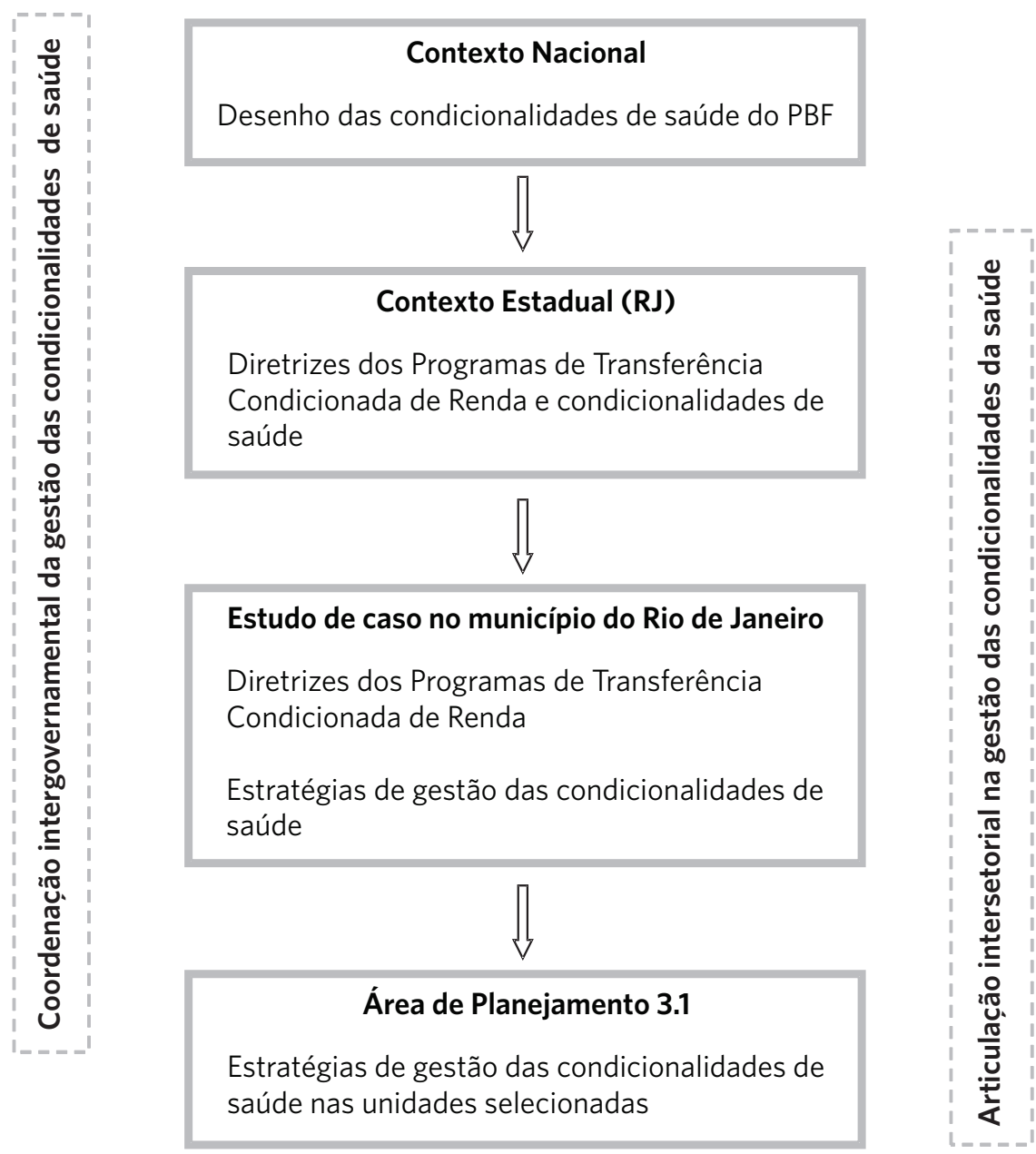

Fonte: Elaboração própria.

A pesquisa compreendeu as seguintes estratégias metodológicas: revisão bibliográfica, análise documental, análise de bases de dados secundários e realização de 20 entrevistas semiestruturadas com atores envolvidos na gestão das condicionalidades de saúde do PBF nas três esferas de governo, selecionados segundo cargo ocupado, atribuição na gestão do programa e tempo de atuação. Os atores selecionados foram distribuídos da seguinte forma: um dirigente do Ministério do Desenvolvimento Social e Combate à Fome (MDS); um ex-dirigente do
MDS; um dirigente do Ministério da Saúde (MS); um dirigente da Secretaria de Estado de Assistência Social e Direitos Humanos (SEASDH); um dirigente da Secretaria Estadual de Saúde (SES); um dirigente da Secretaria Municipal de Desenvolvimento Social (SMDS); um dirigente da Secretaria Municipal de Saúde (SMS); cinco assistentes sociais dos Centros de Referência da Assistência Social (Cras) e oito profissionais das Unidades Básicas de Saúde (UBS) da AP 3.1 do município do Rio de Janeiro.

As entrevistas realizadas compreenderam 
questões relacionadas com a visão dos atores sobre o PBF, o RM e o CFC (programas de transferência condicionada de renda do estado e do município) e as suas condicionalidades de saúde, estratégias de gestão e de articulação adotadas entre os setores. Envolveram ainda perguntas relacionadas com as responsabilidades e atribuições de cada local e esfera de governo, dificuldades encontradas na gestão das condicionalidades de saúde e as estratégias para superá-las. Utilizou-se a técnica de análise de temática das entrevistas, considerando-se as dimensões definidas no estudo.

\section{Resultados}

\section{A gestão nacional das condicionali- dades da saúde do Programa Bolsa Família}

A gestão das condicionalidades da saúde compreende uma rotina complexa, exigindo articulação estreita entre diferentes ministérios, esferas de governo e secretarias envolvidas no processo. A figura 2 apresenta o fluxo de gestão das condicionalidades da saúde.

Figura 2. Fluxo de monitoramento das condicionalidades da saúde

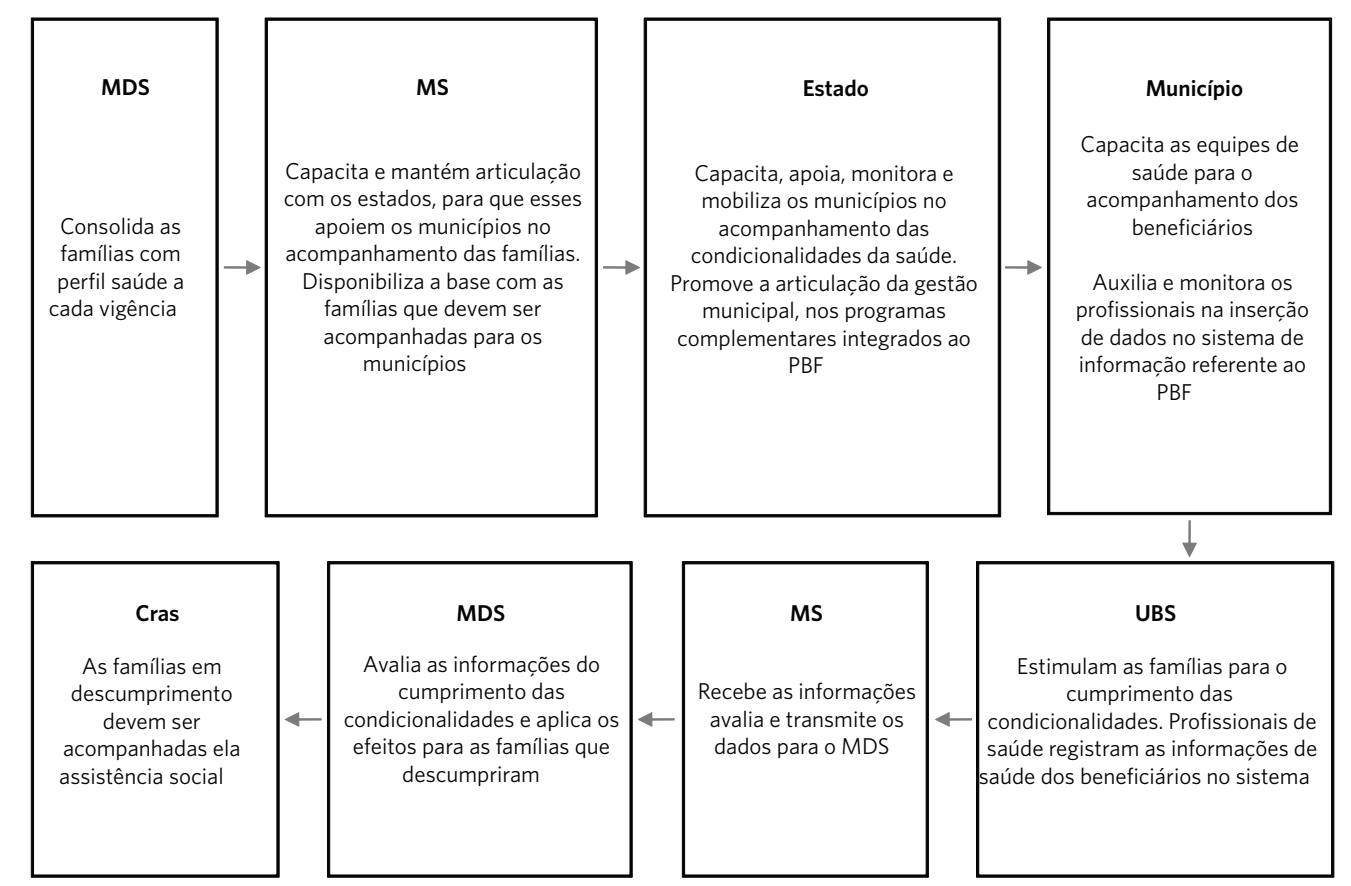

Fonte: Elaboração própria.

Em âmbito nacional, a articulação intersetorial para a gestão do $\mathrm{PBF}$ envolve a realização de reuniões técnicas entre a Coordenação Geral de Alimentação e Nutrição (CGAN) - setor responsável pela gestão das condicionalidades da saúde no
MS - e o MDS, em que são discutidos problemas, prioridades e estratégias.

No que se refere à coordenação intergovernamental, o MS adota estratégias para reforçar a gestão das condicionalidades da saúde, como reuniões técnicas com 
estados e municípios, produção e divulgação de informes sobre o acompanhamento das condicionalidades de saúde; realização de videoconferências com municípios e estados; elaboração de ofícios enviados do nível federal aos estados e municípios; articulação com o Conselho Nacional de Secretarias Municipais de Saúde (Conasems), Conselho das Secretarias Municipais de Saúde (Cosems) e Comissão Intergestores Tripartite (CIT) e oferta do Curso de Educação a Distância sobre o PBF na Saúde, que possibilita a capacitação de diversos profissionais (LIMA, 2013).

Entrevistados citaram três estratégias federais prioritárias para fortalecimento da gestão das condicionalidades de saúde no âmbito local. A primeira seria incentivar os municípios a incorporarem o acompanhamento das condicionalidades de saúde na rotina das atividades da atenção básica. A segunda seria aumentar a identificação precoce das gestantes beneficiárias, visando inseri-las no pré-natal e garantir a concessão do Benefício Variável à Gestante (BVG) no início da gestação. A terceira estratégia seria o monitoramento dos municípios de grande porte, que apresentariam maiores dificuldades na gestão e operacionalização do programa.

Ainda quanto à coordenação intergovernamental, o MS procura estabelecer uma articulação próxima com os estados. Apesar disso, a relação da esfera federal com alguns estados é frágil, sendo variável a adesão dos estados às diretrizes do programa federal, bem como sua capacidade de acompanhamento nas condicionalidades da saúde, como ilustra a tabela 1.

Tabela 1. Percentual de acompanhamento nas condicionalidades da saúde e famílias com perfil saúde por estado. Brasil, segunda vigência de 2013

\begin{tabular}{lrr}
\hline Estado & $\begin{array}{r}\text { \% de famílias totalmente acompanhadas } \\
\text { nas condicionalidades da saúde }\end{array}$ & \begin{tabular}{c} 
Quantidade de famílias com perfil saúde \\
\hline Roraima
\end{tabular} \\
Paraná & 87,6 & 41.305 \\
Tocantins & 80,6 & 373.163 \\
Paraíba & 80,2 & 120.380 \\
Sergipe & 79,8 & 425.429 \\
Rio Grande do Norte & 79,5 & 230.358 \\
Minas Gerais & 79,4 & 303.376 \\
Piauí & 79,2 & 1.016 .159 \\
Ceará & 79,0 & 373.184 \\
Maranhão & 78,2 & 950.825 \\
Alagoas & 77,7 & 824.478 \\
Goiás & 76,9 & 367.336 \\
Pará & 76,6 & 298.961 \\
Bahia & 76 & 743.331 \\
Amazonas & 74,7 & 1.497 .551 \\
Santa Catarina & 74,5 & 303.967 \\
Mato Grosso do Sul & 72,4 & 122.007 \\
\hline
\end{tabular}




\begin{tabular}{lcr}
\hline Tabela 1. (cont.) & & \\
\hline Pernambuco & 71,5 & 953.700 \\
Espírito Santo & 67,2 & 171.374 \\
Mato Grosso & 66,5 & 158.871 \\
Acre & 66,3 & 65.730 \\
São Paulo & 65,8 & 1.097 .421 \\
Rio Grande do Sul & 63,5 & 368.918 \\
Rio de Janeiro & 59,1 & 691.391 \\
Rondônia & 58,3 & 100.313 \\
Amapá & 47,9 & 46.608 \\
Distrito Federal & 32,6 & 76.136 \\
\hline
\end{tabular}

Fonte: Secretaria de Avaliação e Gestão da Informação (Sagi) (BRASIL, 2014).

Existem também conflitos entre o MS e os estados relacionados com o repasse de recursos financeiros que servem como incentivos para serem aplicados na gestão do programa.

Acrescentam-se aos conflitos intergovernamentais as dificuldades de coordenação entre os setores sociais envolvidos na gestão do PBF, incluindo no acompanhamento das condicionalidades que, segundo diversos entrevistados, seriam mais evidentes nos municípios de grande porte. Isso ocorreria pela maior dimensão e complexidade da rede de serviços de saúde e de assistência social, o que exigiria mecanismos de financiamento e de acompanhamento diferenciados.

\section{As condicionalidades da saúde dos Programas de Transferência de Ren- da no estado e no município do Rio de Janeiro}

O estado do Rio de Janeiro apresenta um número expressivo de famílias beneficiárias do PBF, porém um acompanhamento das condicionalidades de saúde relativamente baixo, de aproximadamente $59 \%$ (tabela 1 ).

A análise da gestão das condicionalidades de saúde do PBF na esfera estadual permitiu identificar a importância da atenção básica para a gestão do PBF. A área técnica de alimentação e nutrição - área responsável pelo acompanhamento das condicionalidades da saúde na Secretaria de Estado de Saúde do Rio de Janeiro - possui dificuldades em estabelecer diretrizes para os municípios. Nesse sentido, o envolvimento da equipe estadual de gestão da atenção básica com o PBF foi considerado fundamental para facilitar o envolvimento dos profissionais de saúde dos municípios com o programa.

Os entrevistados da esfera estadual da saúde, assim como os da esfera federal, destacaram a importância de duas estratégias de gestão do PBF relacionadas com as condicionalidades. A primeira seria a capacitação dos municípios quanto aos princípios do programa, inclusive no que diz respeito às condicionalidades de saúde. O objetivo seria evitar ações locais inapropriadas, como a realização de mutirões para monitoramento das condicionalidades que prejudicariam a incorporação do atendimento das famílias na rotina das unidades de saúde e, consequentemente, o acesso. A segunda seria a ampliação da captação precoce das gestantes beneficiárias do $\mathrm{PBF}$ pelos municípios para que elas possam receber o benefício específico no início da gestação.

No aspecto da coordenação intergovernamental, destacam-se acordos de cooperação 
visando à integração do PBF com outros programas nas diferentes esferas governamentais. $\mathrm{O}$ estado do Rio de Janeiro criou o plano Rio Sem Miséria, a partir de pactuação com o plano Brasil Sem Miséria do governo federal. O programa estadual local de transferência de renda - RM - integrava o plano Rio sem Miséria, sendo articulado ao PBF.

O programa RM era coordenado pela SEASDH. Tal programa beneficiava famílias que possuíam renda per capita mensal inferior a $\mathrm{R} \$ 100,00$, utilizando, além da renda declarada registrada no cadastro único, a renda estimada para identificar os beneficiários do programa. $\mathrm{O}$ critério de renda para inclusão no programa estadual em 2014 era, portanto, diferente daquele do PBF, que incluía famílias com renda per capita entre $\mathrm{R} \$ 77,01$ e $\mathrm{R} \$ 154,00$ (desde que possuam crianças e adolescentes) e todas as famílias com renda per capita de até $\mathrm{R} \$ 77,00$.

Ademais, o RM possuía as mesmas condicionalidades do PBF e buscava zerar o hiato de pobreza extrema. Isso porque todas as famílias que já recebiam o benefício do PBF e continuavam com renda per capita familiar inferior a $\mathrm{R} \$ 100,00$ tinham o direito de receber a complementação financeira para superar a linha da pobreza extrema definida pelo estado (CAMPOS FILHO, 2009).

No total, o programa RM estava presente em 91 municípios (todos os municípios do estado, com exceção do município do Rio de Janeiro). A decisão de não implantar o programa estadual no município visaria evitar a sobreposição de programas similares, pois a capital já propusera um programa de transferência de renda próprio, o CFC. Somente o componente do Programa Renda Melhor 'Jovem' foi implantado na capital, por não existir benefício análogo no programa municipal. Se tais decisões, por um lado, sugerem certo esforço de articulação federativa, por outro, os entrevistados apontaram dificuldades na cooperação intergovernamental entre estado e município.

As famílias contempladas pelo programa estadual podiam sacar o benefício do RM pelo cartão do Bolsa Família até o recebimento de um cartão compartilhado (PBF e RM). O compartilhamento do cartão seria relevante para fortalecer a legitimidade do PBF e a cooperação federativa. Além disso, o programa estadual seguia o mesmo calendário de pagamento do programa federal.

$\mathrm{O}$ fato de o RM manter as mesmas condicionalidades do PBF é significativo, pois torna o processo mais integrado, já que, profissionais, gestores e beneficiários estão familiarizados com a metodologia de acompanhamento e monitoramento das contrapartidas. Além disso, assegura um alinhamento com os objetivos nacionais.

A gestão das condicionalidades da saúde do PBF, no município, está sob coordenação da Subsecretaria de Promoção, Atenção Primária e Vigilância em Saúde (SUBPAV). A gestão do PBF pela instância da atenção básica é importante para reforçar a diretriz do cuidado integral em saúde para as famílias beneficiárias.

Em relação à articulação intersetorial entre a saúde e assistência social em nível municipal, observou-se estreita relação entre a SMS e a SMDS, com a realização frequente de reuniões.

O município do Rio de Janeiro, assim como o estado, também possui acordos de cooperação intergovernamental com a União, destacando-se a integração do programa local $\mathrm{CFC}$ ao PBF.

O desenho do CFC foi realizado pela Fundação Getúlio Vargas em conjunto com órgãos do município, como a Casa Civil, o Instituto Pereira Passos, o Instituto de Planejamento, a Secretaria de Assistência Social e a Secretaria Municipal de Educação (NERI, 2010).

Em 2011, houve uma expressiva ampliação do programa, que alcançou $134 \mathrm{mil} \mathrm{fa-}$ mílias assistidas pelo governo municipal (SANTOS; VALE, 2013).

Para ter direito ao $\mathrm{CFC}$, é preciso estar cadastrado no PBF do governo federal, possuir 
renda familiar per capita menor que $\mathrm{R} \$$ 108,00, já incluído o benefício do PBF. Para determinar a renda per capita de cada família, é utilizada a renda estimada, diferentemente do PBF, que utiliza a renda declarada. Buscase zerar o hiato de pobreza extrema, ou seja, todas as famílias que já recebem o benefício do PBF e continuam com renda per capita familiar inferior a $\mathrm{R} \$ 108,00$ (linha de pobreza extrema adotada pelo município do Rio de Janeiro) tem o direito de receber a complementação financeira.

O CFC possuía identidade visual e cartão próprios, não mantendo relação com o cartão do PBF, diferentemente do programa estadual, que adotou um cartão compartilhado.

O CFC também compreendia condicionalidades, mas essas diferiam das contrapartidas do PBF. No que concerne às condicionalidades da educação, é exigido que crianças e adolescentes menores de 18 anos mantenham a frequência mínima de $90 \%$ nas aulas, além da participação de pelo menos um dos responsáveis nas reuniões bimestrais da escola. O não cumprimento da frequência ou a ausência do responsável por dois bimestres consecutivos ocasiona o bloqueio parcial do benefício. A frequência exigida é superior à do $\mathrm{PBF}$, que prevê $85 \%$ para as crianças e adolescentes e $75 \%$ para os estudantes entre 16 e 17 anos. Ressaltase ainda que os alunos que melhorarem seu desempenho escolar ao longo do bimestre recebem um bônus de $\mathrm{R} \$ 50,00$ por bimestre (até $\mathrm{R} \$ 200,00$ por ano).

Esse último aspecto do CFC se distancia dos propósitos das condicionalidades da educação do programa federal, visto que vincula os benefícios financeiros ao desempenho dos alunos, aspecto criticado por entrevistados da esfera federal, dada a maior vulnerabilidade social dessas crianças. As condicionalidades da saúde estabelecidas pelo CFC também são mais específicas e rigorosas, visto que além das informações exigidas pelo PBF, novas obrigatoriedades estão incluídas. Por exemplo, para crianças de 0 a 2 anos, avalia-se a situação de saúde (se teve diarreia, hospitalização ou infecção respiratória aguda no período); para adolescentes, solicita-se participação em um grupo de educação em saúde; e para gestantes, exige-se, no pré-natal, avaliação sobre sífilis e HIV, além de informações sobre o puerpério.

Apesar de serem mais rigorosas, as condicionalidades do CFC têm relação com ações de saúde já desenvolvidas pela atenção básica. Assim, as diferenças das condicionalidades entre os programas nacional e municipal per se não trouxeram dificuldades operacionais adicionais para as equipes de saúde. Os dados oficiais analisados e as entrevistas sugeriram que as unidades de atenção básica que funcionavam com equipes de saúde da família conseguiam ter melhores taxas de acompanhamento das condicionalidades da saúde.

Entretanto, a implantação do CFC ocasionou entraves na rotina do trabalho das assistentes sociais, que, em geral, não conseguiam responder às demandas das famílias relativas ao CFC (as já incluídas ou as que solicitavam inclusão), por não possuírem ingerência sobre o programa.

No contexto local, identificaram-se limites na articulação entre os serviços de saúde e de assistência social na gestão das condicionalidades de saúde. As ações relatadas se resumem a encaminhamentos para o Cras quando as equipes de saúde têm dificuldades. $\mathrm{O}$ insuficiente diálogo entre os setores fragmentava o acompanhamento das condicionalidades da saúde e prejudicava o atendimento à população vulnerável beneficiária dos programas sociais. O quadro 1 sistematiza as principais características dos programas de transferência de renda nacional, estadual e municipal. 


\begin{tabular}{|c|c|c|c|}
\hline Programa & Abrangência & Critérios de inclusão & Condicionalidades da saúde \\
\hline \multirow{3}{*}{ Bolsa Família } & \multirow{3}{*}{ Nacional } & $\begin{array}{l}\text { Famílias com renda per capita de } \mathrm{R} \$ \\
154,00 \text { que possuam crianças e adoles- } \\
\text { centes até } 17 \text { anos }\end{array}$ & $\begin{array}{l}\text { Crianças até } 7 \text { anos: imunização e estado } \\
\text { nutricional }\end{array}$ \\
\hline & & \multirow{2}{*}{$\begin{array}{l}\text { Famílias com renda per capita de } R \$ \\
77,00 \text {, independentemente da composi- } \\
\text { ção familiar }\end{array}$} & $\begin{array}{l}\text { Mulheres de } 10 \text { a } 69 \text { anos: estado nutri- } \\
\text { cional }\end{array}$ \\
\hline & & & Gestantes: pré-natal em dia \\
\hline \multirow{2}{*}{ Renda Melhor } & \multirow{2}{*}{ Estadual } & $\begin{array}{l}\text { Famílias com renda per capita mensal } \\
\text { inferior a R\$ } \\
100,00\end{array}$ & \multirow{2}{*}{ Igual ao PBF } \\
\hline & & $\begin{array}{l}\text { Utiliza a renda declarada e a renda esti- } \\
\text { mada }\end{array}$ & \\
\hline \multirow{4}{*}{$\begin{array}{l}\text { Cartão } \\
\text { Família } \\
\text { Carioca }\end{array}$} & \multirow{4}{*}{ Municipal } & Estar cadastrado no PBF & \multirow[b]{2}{*}{$\begin{array}{l}\text { Crianças de } 0 \text { a } 2 \text { anos: imunização, } \\
\text { estado nutricional, tipo de aleitamento e } \\
\text { situação de saúde }\end{array}$} \\
\hline & & $\begin{array}{l}\text { Famílias com renda per capita mensal } \\
\text { inferior a } R \$ \\
108,00\end{array}$ & \\
\hline & & \multirow{2}{*}{ Utiliza a renda estimada } & $\begin{array}{l}\text { Adolescentes de } 12 \text { a } 19 \text { anos: imunização } \\
\text { e grupo de educação em saúde }\end{array}$ \\
\hline & & & $\begin{array}{l}\text { Gestantes: imunização, pré-natal em dia } \\
\text { e puerpério }\end{array}$ \\
\hline
\end{tabular}

Fonte: Elaboração própria.

No que se refere ao atendimento das famílias do programa, constatou-se que raramente ocorria uma abordagem integral, o que se distancia da diretriz nacional do PBF. Os profissionais de saúde priorizavam o registro das informações referentes às condicionalidades para as gestantes e crianças, o que limita a atuação dos serviços para as famílias do programa.

Observaram-se dificuldades de compreensão dos profissionais de saúde sobre o significado e a relevância do programa e das condicionalidades de saúde. Alguns profissionais baseavam-se em argumentos de risco moral, como o possível comportamento oportunista dos pobres. Entre os profissionais da assistência social, a maioria ressaltou a importância do programa e do seu objetivo. Todavia, mesmo nesse grupo, houve entrevistados que enfatizaram a suposta dependência financeira e acomodação dos pobres em função do benefício do programa.

Por último, um aspecto importante destacado pela maioria dos dirigentes e profissionais da área de assistência social entrevistados diz respeito à insuficiência de recursos humanos e à precária infraestrutura para dar conta das responsabilidades no acompanhamento das famílias.

\section{Discussão}

O estudo identificou fragilidades na articulação intergovernamental e na coordenação intersetorial da gestão das condicionalidades da saúde do PBF, que prejudicam o alcance dos propósitos das condicionalidades enunciados nos documentos oficiais do programa nacional, de contribuir para o exercício do direito à saúde e consequente interrupção do ciclo de pobreza.

No que concerne à coordenação intergovernamental, identificaram-se dificuldades relacionadas com o financiamento, com a cooperação e articulação entre as esferas e com diferentes concepções sobre os propósitos do programa e das condicionalidades. 
No âmbito do financiamento, observaram-se conflitos relacionados com o mecanismo de definição dos repasses financeiros para a gestão do PBF, que é adotado como incentivo à melhoria da gestão estadual e municipal. Outro estudo também apontou tais conflitos, apontando que a baixa cobertura das condicionalidades da saúde limitaria o montante dos repasses mensalmente (MONNERAT, 2009).

Em virtude desses mecanismos, há riscos de os governos subnacionais serem complacentes com informações indevidas sobre as condicionalidades a fim de obter mais recursos (LINDERT, 2007).

No que diz respeito aos acordos de cooperação, os governos subnacionais possuem considerável autonomia para formular e implementar inovações no desenho dos programas locais integrados ao PBF. O programa estadual RM mostrou-se consoante com o programa federal ao utilizar um cartão compartilhado para realizar o saque do benefício, seguir o mesmo calendário de pagamento e, especialmente, manter as mesmas condicionalidades do PBF.

Em contraposição, o programa municipal CFC utiliza um cartão específico para o pagamento do benefício e optou por implantar condicionalidades adicionais tanto na área da educação como na da saúde. Adotou-se uma lógica comportamental no desenho das condicionalidades, muito evidente na área educacional, ao transferir responsabilidades para as famílias e reforçar exigências e mecanismos de punição ou premiação vinculados ao comportamento dos indivíduos.

Defensores argumentam que essa agenda de premiar os alunos avaliando o seu desempenho está em concordância com a ideia de que os pobres devem ser motivados por incentivos, e não penalizados; e que a presença de condicionalidades mais rigorosas no CFC poderia abrir as portas do mercado de trabalho para as famílias mais vulneráveis (NERI, 2010). Entretanto, estratégias mais abrangentes como o investimento na qualidade do ensino público e a formulação de políticas de emprego adequadas para assegurar melhores oportunidades de inserção dessas famílias no mercado de trabalho poderiam ser mais efetivas para esses propósitos.

No que concerne à articulação intersetorial, o estudo também apontou fragilidades, especialmente no contexto local, nas relações entre as áreas de saúde e assistência social, expressas na limitada comunicação entre os setores e na escassez de iniciativas de acompanhamento integrado das famílias beneficiárias. Tais limitações restringem a possibilidade de o acompanhamento das condicionalidades servir como estratégia para identificar famílias em situação de maior vulnerabilidade e apoiá-las por meio de estratégias abrangentes voltadas a sua inserção social.

Conclui-se que a coordenação intergovernamental e intersetorial na gestão das condicionalidades da saúde do PBF, somada aos requisitos de articulação com os programas de transferência de renda subnacionais (no caso em questão, o RM e o CFC), constitui tarefa complexa, visto que depende do envolvimento, mobilização e articulação de um grande número de setores e atores presentes nas esferas federal, estadual e municipal.

Além das limitações nas relações intersetoriais e intergovernamentais, a pesquisa sugeriu que as ações relacionadas com as condicionalidades de saúde destinadas aos beneficiários dos programas são precárias, restringindo-se principalmente ao registro administrativo das informações, aspecto também evidenciado por Trevisani (2007).

Se a articulação entre estratégias de garantia de direitos sociais e de combate à pobreza em uma perspectiva intersetorial e intergovernamental não for assegurada, os PTRC podem se tornar mecanismos isolados de cumprimento das metas governamentais, amenizando em curto prazo situações insatisfatórias, porém distanciando-se da sua proposta de alterar as condições de pobreza em longo prazo (FONSECA; VIANA, 2007).

Ressalte-se ainda a dificuldade de 
compreensão e o posicionamento crítico dos profissionais entrevistados - principalmente os de saúde - não apenas em relação às condicionalidades, mas quanto ao próprio significado e relevância dos programas de transferência de renda.

Estudos sobre o PBF refutam argumentos mencionados pelos profissionais, como acomodação dos beneficiários, por exemplo, no que se refere às condições de trabalho ou remuneração (BARBOSA; CORSEUIL, 2013; JANUZZI; PINTO, 2013). Ao contrário, sugerem que o programa tem repercussões relevantes além do aumento da renda, como a maior autonomia das mulheres beneficiárias (REGO; PINZANI, 2013). Outros refutam o argumento que o programa induziria as mulheres a terem mais filhos, ao apontarem a diminuição da fecundidade entre as mulheres que recebem o benefício (ALVEZ; CAVENAGHI, 2013).

O sucesso de um programa de transferência condicionada de renda, no que concerne à melhoria das condições sociais e de saúde de famílias beneficiárias, dependeria da existência de serviços de saúde eficazes e de uma boa infraestrutura local dos serviços sociais, o que apresentou limitações no estudo (LAGARDE; HAINES; PALMER, 2007).

Neste estudo, a atenção básica foi destacada como fundamental na gestão das condicionalidades da saúde do PBF em todas as esferas. No contexto local, observou-se o maior acompanhamento das famílias por unidades que possuem todo o território coberto pelas equipes de saúde da família. Esses resultados sugerem o potencial de articulação positiva entre a Estratégia Saúde da Família e o PBF. Tais programas federais têm como características comuns: desenho federal, com diretrizes de descentralização e intersetorialidade; foco na família como unidade de atenção e cuidado; cobertura nacional ampla e muito expressiva na população mais vulnerável e pobre. Além disso, as condicionalidades da saúde do PBF fazem parte do escopo de ações e serviços sob responsabilidade das equipes de saúde da família, o que endossa a necessidade de articulação entre os programas.

Destaque-se que as ações englobadas pelas condicionalidades da saúde são extremamente básicas (imunização, acompanhamento nutricional e realização do pré-natal) e já deveriam ser asseguradas a toda a população. Nesse sentido, cabem questionamentos quanto à pertinência de exigi-las como contrapartida ao recebimento de transferências monetárias em um país como o Brasil, cujo sistema de saúde já deveria assegurar acesso público e universal de toda população aos diversos níveis de atenção.

No caso brasileiro, o mais relevante seria enfrentar as barreiras geográficas e organizacionais de acesso da população mais pobre aos serviços públicos de saúde, bem como melhorar a qualidade do cuidado ofertado em todos os níveis de atenção (ESTRELLA; RIBEIRO, 2008).

Em termos de agenda de pesquisa, seria importante desenvolver estudos mais específicos sobre os efeitos das condicionalidades da saúde. Vários efeitos positivos dos PTRC na educação têm sido documentados. $\mathrm{O}$ aumento da matrícula escolar, a diminuição na taxa de abandono e da taxa de repetição foram documentados por Glewwe e Kassouf (2012). O aumento da frequência escolar foi referido por Bastagli (2008). Já foram sugeridos também resultados positivos em longo prazo no desenvolvimento cognitivo e socioemocional dos beneficiários (PAXSON; SCHADY, 2007). Contudo, estudos sobre as condicionalidades da saúde são escassos, não havendo evidências substantivas de seus efeitos positivos no caso brasileiro até o momento. Seria interessante analisar se a Estratégia Saúde da Família, articulada ao PBF, poderia ampliar o acesso à saúde das famílias beneficiárias. Outra questão a ser investigada seria se e como a atenção nos serviços de saúde poderia contribuir para melhorar as condições de vida dessas famílias. 


\section{Conclusões}

A existência de condicionalidades no PBF é justificada, nos documentos oficiais, com o propósito de favorecer o acesso a direitos sociais básicos de saúde e educação para romper com o ciclo intergeracional da pobreza nas famílias beneficiárias. No entanto, o estudo sugere que a exigência e o acompanhamento das contrapartidas relacionadas com ações de saúde per se não são suficientes para o alcance desse propósito. É fundamental avançar na reorganização dos serviços e no envolvimento dos profissionais, para que as ações de saúde e sociais sejam ofertadas de forma articulada e com qualidade paras as famílias beneficiárias.

Os programas de combate à pobreza devem estar conjugados com políticas distributivas e de caráter estrutural, como forma de promover um desenvolvimento mais igualitário. Isso requer o fortalecimento de políticas sociais abrangentes com base na prestação de serviços universais de qualidade, como nas áreas de educação e saúde. No último caso, um caminho mais promissor do que a ampliação e gerenciamento de condicionalidades para as famílias pobres que recebem benefícios monetários seria avançar na expansão dos serviços públicos, na redução das desigualdades e na consolidação do SUS.

\section{Colaboradores}

MORAES, V. D. Participou da concepção do artigo, levantamento, processamento e análise dos dados e da redação do artigo.

MACHADO, C. V. Participou da concepção do artigo, análise dos resultados, redação e revisão final do artigo.

\section{Agradecimentos}

Verena Duarte de Moraes é bolsista de doutorado da Coordenação de Aperfeiçoamento de Pessoal de Nível Superior (Capes) e Cristiani Vieira Machado bolsista de produtividade em pesquisa do Conselho Nacional de Desenvolvimento Científico e Tecnológico (CNPq).

\section{Referências}

ALVES, J. E. D.; CAVENAGHI, S. O Programa Bolsa

Família e as taxas de fecundidade no Brasil. In: CAMPELLO, T.; NERI, M. C. (Org.). Programa Bolsa

Família: uma década de inclusão e cidadania. Brasília, DF: IPEA, 2013. p. 233-245.

BARBOSA, A. L. N. H.; CORSEUIL, C. H. L. Bolsa Família, escolha ocupacional e informalidade no Brasil. In: CAMPELLO, T.; NERI, M. C. (Org.). Programa Bolsa
Família: uma década de inclusão e cidadania. Brasília, DF: IPEA, 2013. p. 327- 339.

BASTAGLI, F. The design, implementation and impact of conditional cash transfers targeted on the poor: An evaluation of Brazil's Bolsa Família. Working paper London: London School of Economics and Political Science (University of London), 2008. Disponível em: <http://ethos.bl.uk/OrderDetails.do?uin=uk. 
bl.ethos.540368>. Acesso em: 15 jun. 2017

BRASIL. Ministério do Desenvolvimento Social. Secretaria de Avaliação e Gestão da Informação. Disponível em: <http://aplicacoes.mds.gov.br/sagi/portal/>. Acesso em: 10 out. 2014.

Ministério do Desenvolvimento Social e Combate à Fome (MDS). Bolsa Família: Cidadania e Dignidade para Milhões de Brasileiros. Brasília, DF: MDS, 2010. Disponível em: <http://dataspace.princeton.edu/jspui/handle/88435/dsp0ljq085n248>. Acesso em: 15 jun. 2017

CAMPOS FILHO, A. C.; VIEIRA, A. M. M.; MARTINS, L. G. Rio sem Miséria: uma experiência de cooperação federativa para a superação da pobreza extrema. Debates Sociais, Rio de Janeiro, n. 71-72, p. 191-202, 2009.

DRAIBE, S. M. Uma nova agenda social na América Latina? Pontos de partida para a análise comparada dos sistemas de proteção social e suas mudanças recentes. In: SOLA, L.; LOUREIRO, M. R. G. (Org.). Democracia, mercado e Estado: o B de Brics. Rio de Janeiro: FGV, 2011. p. $249-288$.

Uma nova institucionalidade das políticas sociais? Reflexões a propósito da experiência latino-americana recente de reforma dos programas sociais. São Paulo em perspectiva, São Paulo, v. 11, n. 4, p. 3-15, 1997. Disponível em: <https://edisciplinas.usp.br/pluginfile. php/2092019/mod_resource/content/1/Draibe\%20 politicas\%20p\%C3\%BAblicasv11n04_01.pdf>. Acesso em: 12 mar. 2015.

ESTRELLA, J.; RIBEIRO, L. M. Qualidade da gestão das condicionalidades do Programa Bolsa Família: uma discussão sobre o índice de gestão descentralizada. Rev. Adm. Pública, Rio de Janeiro, v. 42, n. 3, p. 625-641, jun. 2008. Disponível em: <http:// www.scielo.br/scielo.php?script=sci_arttext\&pid $=$ S0034-76122008000300009 $>$. Acesso em: 14 jun. 2015 .

FONSECA, A. M. M.; VIANA, A. L. A. Direito à saúde, atenção básica e transferências condicionadas de renda na América Latina. Ciência \&t Saúde Coletiva, Rio de Janeiro, v. 12, n.6, p. 1505-1512, dez. 2007. Disponível em: <http://www.scielo.br/scielo.php?pid=S141381232 $007000600012 \&$ script $=$ sci_abstract $\&$ tlng $=$ pt $>$. Acesso em: 20 out. 2015

GLEWWE, P.; KASSOUF, A. L. The impact of the Bolsa Escola/Familia conditional cash transfer program on enrollment, dropout rates and grade promotion in Brazil. Journal of Development Economics, Amsterdam, v. 97, n. 2, p. 505-517, mar. 2012. Disponível em: <http://www.anpec.org.br/encontro2008/artigos/200807211140170-.pdf>. Acesso em: 12 dez. 2015.

JANNUZZI, P. M.; PINTO, A. R. Bolsa Família e seus impactos nas condições de vida da população brasileira: uma síntese dos principais achados da pesquisa de avaliação de impacto do Bolsa Família II. In: CAMPELlO, T.; NERI, M. C. (Org.). Programa Bolsa Família: uma década de inclusão e cidadania. Brasília, DF: IPEA, 2013. p. 179-192.

JANVRY, A.; FINAN, F.; SADOULET, E. Local governance and efficiency of conditional cash transfers: Bolsa Escola in Brazil. University of California, Berkeley. p. 1-35, set. 2007. Disponível em: <http://eml. berkeley.edu/ webfac/bardhan/e271_f07/bolsa.pdf>. Acesso em: 15 jun. 2017

\section{LAGARDE, M.; HAINES, A.; PALMER, N.}

Conditional cash transfers for improving uptake of health interventions in low-and middle-income countries: a systematic review. Jama, Chicago, v. 298, n. 16, p. 1900-1910, out. 2007. Disponível em: <http://epri.org.za/wpcontent/uploads/2011/03/ Lagardeetal2007ConditionalCashTransfersl.pdf $>$. Acesso em: 15 mar. 2014

LAVINAS, L. 21st Century Welfare. London: New Left Review, 2013. Disponível em: <https://newleftreview. org/II/84/lena-lavinas-21st-century-welfare>. Acesso em: 20 abr. 2016.

LIMA, A. M. C. O desempenho do setor saúde no acompanhamento das condicionalidades do Programa Bolsa Família: a intersetorialidade em ação. 2013. 76 f. Dissertação (Mestrado em Saúde Pública) - Escola 
Nacional de Saúde Pública Sergio Arouca, Fundação Oswaldo Cruz, Rio de Janeiro, 2013. Disponível em: $<$ http://saudepublica.bvs.br/pesquisa/resource/pt/ens30591>. Acesso em: 13 dez. 2016.

LINDERT, K. et al. The nuts and bolts of Brazil's Bolsa Família Program: implementing conditional cash transfers in a decentralized context. Protection Discussion Paper. Series of World Bank Working Papers, n. 709, p. 1-144, maio 2007. Disponível em: <http://siteresources.worldbank.org/INTLACREGTOPLABSOCPRO/ Resources/BRBolsaFamiliaDiscussionPaper.pdf $>$. Acesso em: 11 nov. 2015.

MACHADO, C. V. Desenvolvimento, proteção social e política de saúde: o Brasil no contexto latino-americano. In: VIANA, A. L. D.; BOUSQUAT, A.; IBAÑEZ, N. (Org.). Saúde desenvolvimento, ciência, tecnologia e inovação. São Paulo: Hucitec, 2012. p. 65-98.

MEDEIROS, M.; BRITTO, T.; SOARES, F. Transferência de renda no Brasil. Novos estud. CEBRAP, São Paulo, n. 79, p. 5-21, nov. 2007. Disponível em: <http://www.scielo.br/scielo.php?script=sci_artt ext\&pid=S010133002007000300001>. Acesso em: 30 maio 2015 .

MONNERAT, G. L. Transferência condicionada de renda, saúde e intersetorialidade: lições do Programa Bolsa Família. 2009. 283 f. Tese (Doutorado em Saúde Pública) - Escola Nacional de Saúde Pública Sergio Arouca, Fundação Oswaldo Cruz, Rio de Janeiro, 2009. Disponível em: <http://bvssp.icict.fiocruz.br/ pdf/25496_Giselle_Lavinas_Monnerat.pdf>. Acesso em: 19 ago. 2014.

NERI, M. Cartão Família Carioca: o Bolsa Família 2.0. Revista Conjuntura Econômica, Rio de Janeiro, v. 64, n. 11, p. 60-64, jan. 2010. Disponível em: <http:// bibliotecadigital.fgv.br/ojs/index.php/rce/article/ view/24133/22912>. Acesso em: 28 maio 2016.
PAXSON, C.; SCHADY, N. Does Money Matter? The Effects of Cash Transfers on Child Health and Development in Rural Ecuador. Economic Development and Cultural Change [S. 1.], v. 59, n. 1, p. 187-229, out. 2010.

REGO, W. G. D. L.; PINZANI, A. Vozes do Bolsa Família: autonomia, dinheiro e cidadania. São Paulo: Unesp, 2013.

\section{SANTOS, V. C.; VALE, M. R. O Programa Cartão}

Família Carioca como Expressão Contemporânea do Processo de Assistencialização. In: Jornada Internacional de Políticas Públicas, 6, 2013, Maranhão. Anais eletrônicos... 2013. Disponível em: <http://www.joinpp.ufma.br/jornadas/joinpp2013/ JornadaEixo2013/anais-eixo5-pobrezaepoliticaspublicas/oprogramacartaofamiliacariocacomoexpressaocontemporaneadoprocessodeassistencializacao.pdf $>$. Acesso em: 3 out. 2014.

SIMÕES, A. A. The contribution of Bolsa Família to the educational achievement of economically disadvantaged children in Brazil. 2012. 277 f. Tese (Doutorado em Filosofia) - Universidade de Sussex, Brighton, 2012. Disponível em: <http://sro.sussex.ac.uk/40673/1/ Sim\%C3\%B5es\%2C_Armando_Amorim.pdf>. Acesso em: 15 jun. 2017.

TREVISANI, J. J. D. Avaliação da Implementação das condicionalidades de saúde do Programa Bolsa Família e seu papel no cuidado à saúde: estudo de caso do município do Rio de Janeiro. 2012. 193 f. Tese (Doutorado em Nutrição em Saúde Pública) - Universidade de São Paulo, São Paulo, 2012. Disponível em: <http://www. teses.usp.br/teses/disponiveis/6/6138/tde-30052012134711/pt-br.php>. Acesso em: 10 jun. 2016.

Recebido para publicação em fevereiro de 2017

Versão final em junho de 2017

Conflito de interesses: inexistente

Suporte financeiro: apoio financeiro do CNPq (Edital Universal 2013) com o processo de número: 474502/2013-9 